\title{
Nervus intermedius dysfunctions after vestibular schwannoma surgery: a prospective clinical study
}

\author{
Hussam Metwali, MD, MSc, Katja Kniese, MD, Babak Kardavani, MD, Venelin Gerganov, MD, PhD, \\ and Madjid Samii, MD, PhD
}

International Neuroscience Institute, Hannover, Germany

OBJECTIVE The purpose of this prospective study was to evaluate the dysfunction of the nervus intermedius ( $\mathrm{NI})$ after vestibular schwannoma (VS) surgery. The authors present a clinically feasible method for this purpose.

METHODS In this prospective study, the authors included 30 patients who underwent surgery at the International Neuroscience Institute between May 2014 and February 2017 for resection of VS. The patients' taste sensation was examined using taste strips. Lacrimation was tested using the Schirmer I test. The clinical evaluation was performed before surgery and repeated at 2 weeks and at 6 months after surgery as well as during the follow-up, which extended up to 2 years. The authors tested the correlation between the $\mathrm{NI}$ dysfunctions and the House-Brackmann grade of facial nerve palsy.

RESULTS The taste sensation was lost on the side of surgery in 2 patients (6.6\%) and decreased in 4 patients (13.3\%). The disturbance of taste sensation was not statistically correlated with dysfunctions of the motor portion of the facial nerve. The taste impairment resolved in 4 patients within 6 months, but 2 patients suffered from persistent loss of the taste sensation on the side of surgery during the follow-up. In 23 patients (76.6\%), the baseline lacrimation was lower on the side of surgery, and it was significantly correlated with outcome for dysfunctions of the motor portion of the facial nerve. During the follow-up, baseline lacrimation improved in correlation with the improvement in the dysfunctions of the motor portion of the facial nerve. None of the patients reported change in salivation or nasal secretion.

CONCLUSIONS The NI can be affected after VS surgery. The disturbance of baseline lacrimal secretion was correlated with dysfunctions of the motor portion of the facial nerve. However, the disturbance of the taste sensation was not correlated with the grade of facial nerve palsy. Dysfunctions of the NI should be evaluated and separately reported while analyzing facial nerve outcome after VS surgery.

https://thejns.org/doi/abs/10.3171/2018.4.JNS1818

KEYWORDS facial nerve; vestibular schwannoma; outcome; cerebellopontine angle

$\mathrm{P}$ RESERVATION of facial nerve function has become one of the main challenges of the resection of vestibular schwannoma (VS). Most of the reports about the surgical outcome of this procedure focus on the motor function of the facial nerve.,17-19 The nervus intermedius (NI) - the sensory/parasympathetic part of the facial nerve-has gained less attention, however, during the evaluation of the postoperative outcome.22

The nervus intermedius, also known as the nerve of Wrisberg, was first described by Heinrich August Wrisberg in 1563, and it was named the "portio media inter co- municantem faciei et nervum auditorium." ${ }^{3}$ It conveys the taste sensation from the anterior two-thirds of the tongue and parasympathetic secretory fibers via the great petrosal nerve to the lacrimal gland, and via the corda tympani to the submandibular and sublingual salivary glands. It is responsible also for parasympathetic innervation to the nasal mucosa. Furthermore, it carries afferent fibers of sensory perception from the skin of the external auditory meatus, nasopharynx, and nose. ${ }^{2,15}$

Symptoms related to NI dysfunctions have been reported after microsurgical or radiosurgical management

ABBREVIATIONS HB = House-Brackmann; $\mathrm{NI}=$ nervus intermedius; $\mathrm{VS}=$ vestibular schwannoma

SUBMITTED January 4, 2018. ACCEPTED April 2, 2018.

INCLUDE WHEN CITING Published online September 7, 2018; DOI: 10.3171/2018.4.JNS1818. 
of VS. ${ }^{14,20,24}$ Some studies depended on self-assessment questionnaires mailed to the patients. ${ }^{14,20}$ The taste sensation in cases of VS could be objectively tested using taste strips ${ }^{4,9}$ or solutions. ${ }^{16}$ It could also be tested using electrogustometry. ${ }^{24}$

In this study, we performed a prospective clinical analysis of the pre- and postoperative functions of the nonmotor portion of the facial nerve, specifically the taste and lacrimation functions in patients who underwent the resection of VS at our institute. We correlated the dysfunction of the nonmotor portion of the facial nerve with the grade of postoperative facial nerve palsy. We also present the long-term follow-up of the deficits related to NI. To the best of our knowledge, this is the first prospective clinical study to use objective testing of the NI dysfunction after VS surgery. Our study could help in preoperative patient counseling and it illuminates other parameters to report the quality of life and outcome after VS surgery. The possibilities of recovery from the NI deficits have also been explained.

\section{Methods}

This study has been approved by the local ethics committee of Hannover Medical School. Between May 2014 and February 2017, we performed a prospective analysis of the taste and lacrimation functions before and after VS surgery in 30 patients. The clinical evaluation was performed before surgery, 2 weeks after surgery, 6 months after surgery, and during the follow-up visits. The earliest postoperative evaluation was performed 2 weeks after surgery to avoid any possible confounding effects of anesthesia or postoperative medications. The follow-up visits were after 6 months in 30 patients, 1 year in 26 patients, and 18 months in 22 patients. Twenty patients (including the individuals with delayed recovery from the NI dysfunctions) were followed for 2 years. We excluded cases of neurofibromatosis, recurrence, previous surgery, or radiation.

\section{Examination of the Taste Sensation}

The patients were asked to avoid smoking or eating 2 hours before the test. We examined the taste sensation of the anterior two-thirds of the tongue by using taste strips (Burghard Messtechnik).,9 The strips had the taste of salt, sweet, and acidic. We applied the strips sequentially to the side of the tongue. Between the application of different taste strips, the patient was asked to wash her/his mouth with water. The patient was asked to recognize the taste modality and compare the intensity to that perceived on the normal side. Each patient received points ranging from 0 to 3 ( 0 denoted preservation of the three modalities and 3 meant loss of the three modalities). During the postoperative control and the follow-up visits, the patients were asked to report about the subjective feeling of altered or deficient taste sensation.

\section{Examination of Lacrimation}

Local anesthetic was applied to both eyes $(0.5 \%$ proparacaine hydrochloride eye drops) before the test to evaluate the baseline lacrimal secretion without reflex secre- tion due to conjunctival irritation (Schirmer I test).${ }^{10}$ Strips of test papers (Schirmer Mark Blu; Optitech Eyecare) were inserted in the inferior fornix of both eyes. We asked the patient to close the eyes gently. The test took 5 minutes. The paper strips were removed from both eyes, and the length of the moistened part of the strip was measured in millimeters. The length can be easily recognized due to the blue coloration of the wet strips. To avoid individual variations, we used the differences (in $\mathrm{mm}$ ) between the side of surgery and the normal side for further statistical analysis. The patients were also asked to report the feeling of dry eye.

\section{Salivation and Nasal Secretion}

Our patients were asked to report changes in salivation or nasal secretion before surgery and 2 weeks after surgery. During the 6-month follow-up visit, the patients were asked if they had noticed changes in salivation or nasal secretion anytime during the postoperative period up until that point.

\section{Clinical Outcome}

The clinical outcome was evaluated in this study according to the appearance of new deficits or the worsening of preexisting deficits after surgery. The outcome for dysfunctions of the motor portion of the facial nerve was reported according to the House-Brackmann (HB) grading system. ${ }^{7}$

\section{Results}

\section{Patient Characteristics}

This group of patients included 17 men and 13 women. The ages in the group ranged from 23 to 61 years (average 42 years).

\section{Tumor Size}

The size of the VS was reported according to the Hannover classification: T1, intracanalicular growth only; T2, intra- and extracanalicular growth pattern with a tumor in the cerebellopontine angle; T3, intra- and extracanalicular growth pattern with brainstem contact; and T4, intra- and extracanalicular growth pattern with compression of the brainstem. ${ }^{18}$ In 9 patients the tumor size was T2, in 13 patients it was T3, and in 8 patients it was T4 (Table 1).

\section{Tumor Resection and Operative Findings}

All patients were surgically treated via the retrosigmoid transmeatal approach. Complete tumor resection was achieved in 29 patients. In the remaining patient, the tumor was very adherent to the facial nerve, and a thin sheath of the lesion was left on the nerve. The continuity of the facial nerve was anatomically preserved in all cases, and this could be confirmed by electrical stimulation.

\section{Outcome for the Motor Portion of the Facial Nerve}

None of our patients showed facial nerve palsy preoperatively. Two weeks after surgery, 9 patients had normal functions of the motor portion of the facial nerve; 14 patients had Grade II facial palsy, 6 had Grade III, and 1 had 
TABLE 1. Summary of characteristics in 30 patients with VS

\begin{tabular}{lc}
\hline \multicolumn{1}{c}{ Characteristic } & Value \\
\hline No. of patients & 30 \\
\hline Age range & 23-61 yrs (average 42 yrs) \\
\hline Male/female ratio & $17: 13$ \\
\hline Tumor size* & \\
\hline T1 & 0 \\
\hline T2 & 9 \\
\hline T3 & 13 \\
\hline T4 & 8 \\
\hline
\end{tabular}

Values represent the number of patients unless otherwise indicated.

${ }^{*}$ According to the Hannover classification (Samii and Matthies).

Grade IV. After 6 months, 23 patients regained normal functions of the motor portion of the facial nerve. However, 6 patients showed HB Grade II facial nerve palsy and 1 patient had HB Grade III. The patients with HB Grade II facial nerve palsy showed further improvement until they achieved normal facial nerve functions after a period of 12-18 months. The patient who initially had HB Grade IV postoperative facial nerve palsy reached Grade II after 24 months.

\section{Outcome for the Nonmotor Portion of the Facial Nerve Taste Sensation in the Early Postoperative Period}

Preoperatively, 1 patient showed decreased taste sensation on the side of the tumor and remained unchanged after the surgery, but resolved after 6 months. Postoperatively, 2 patients lost taste sensation on the side of surgery (for salt, sweet, and sour). One of them had normal functions of the motor portion of the facial nerve postoperatively, and the other had HB Grade III facial palsy (Table 2). Both recognized subjectively the lost taste sensation. Four patients, however, showed new disturbed taste functions (markedly decreased intensity compared to the healthy side and/or failure to recognize taste modality) on the side of surgery after tumor resection. Only 2 of them could subjectively recognize the decreased taste. Three patients, including 2 of the patients with decreased taste sensation, reported a strange (metallic) taste in the mouth.

Long-Term Follow-Up of the Taste Sensation

After 6 months, the group of patients who experienced impaired taste sensation or a strange taste after surgery (6 patients) recovered completely, including the patient with preoperatively impaired taste sensation. The patients with lost taste sensation did not recover after a 2-year follow-up period. They also reported that they subjectively lost the taste sensation on the side of surgery.

\section{Lacrimation in the Early Postoperative Period}

In 2 patients there was a difference preoperatively in baseline lacrimation between both eyes $(2 \mathrm{~mm}$ lower on the side of the tumor). However, they did not suffer from the symptom of dry eye. In 23 patients, lacrimation on the affected side was lower postoperatively than on the healthy side (mean $7.2 \mathrm{~mm}$, SD $1.9 \mathrm{~mm}$ ). All patients with decreased lacrimation on Schirmer's test reported the subjective feeling of dry eye. Four patients have reported early postoperative epiphora (an overflow of tears onto the face). Three of them had Grade III facial palsy, and 1 had Grade IV facial palsy (Table 2). The baseline lacrimation on the healthy side did not change after surgery; thus we used that side as a reference for comparison. In this series, the decreased lacrimation was on the side of surgery in all affected patients. None of the patients showed higher baseline lacrimation on the side of surgery.

\section{Long-Term Follow-Up of Lacrimation}

After 6 months, the symptoms of dry eye resolved and there was no difference between sides with Schirmer's test in 20 patients. Only 3 patients reported the persistent feeling of dry eye and positive Schirmer I test (decreased moisture on the side of surgery) in the follow-up. One of them had HB Grade III facial nerve palsy, and 2 had HB Grade II. Two patients with improving Grade II facial palsy recovered from the symptoms of dry eye after 1 year. The patient with HB Grade III facial palsy showed further improvement after 24 months regarding the facial palsy, which reached Grade II, but was still reporting a subjective feeling of dry eye. However, Schirmer's tested showed equal baseline lacrimation in both eyes.

One patient reported experiencing crocodile tears 2 months after surgery. This patient did not show postoperative dysfunction of the motor portion of the facial nerve, and the symptoms resolved after 6 months. The postoperative epiphora resolved in 3 patients within 6 months, and in the fourth patient after 1 year.

TABLE 2. Summary of the outcome for the motor portion of the facial nerve, taste disturbance, and lacrimation 2 weeks after surgery

\begin{tabular}{ccccc}
\hline $\begin{array}{c}\text { HB Grade of Facial } \\
\text { Nerve Palsy }\end{array}$ & $\begin{array}{c}\text { No. of } \\
\text { Patients }\end{array}$ & $\begin{array}{c}\text { No. of Patients } \\
\text { w/ Lost Taste }\end{array}$ & $\begin{array}{c}\text { No. of Patients } \\
\text { w/ Decreased Taste }\end{array}$ & $\begin{array}{c}\text { Mean Diff Btwn Side of Op \& Healthy } \\
\text { Side According to Schirmer's Test }\end{array}$ \\
\hline I & 9 & 1 & 0 & $0.8 \mathrm{~mm}(0-2 \mathrm{~mm})$ \\
II & 14 & 0 & 2 & $3.3 \mathrm{~mm}(0-6 \mathrm{~mm})$ \\
III & 6 & 1 & 2 & $6.5 \mathrm{~mm}(5-8 \mathrm{~mm})$ \\
IV & 1 & 0 & 0 & $8 \mathrm{~mm}$ \\
\hline $\mathrm{V}$ & 0 & NA & NA & $\mathrm{NA}$ \\
\hline $\mathrm{VI}$ & 0 & NA & NA & $\mathrm{NA}$ \\
\hline
\end{tabular}

Diff = difference; $N A=$ not applicable. 


\section{Effect on Salivation and Nasal Secretion}

None of the patients in this series reported changes in salivation or nasal secretion in the early or late postoperative period.

\section{Other Cranial Nerve Dysfunctions}

One patient suffered from mild facial hypesthesia with preserved corneal reflex before surgery, which improved in the follow-up examination after surgery. There was no new effect on the trigeminal nerve. None of the patients in this series suffered from lower cranial nerve deficits.

\section{Statistical Analysis}

The facial nerve palsy was analyzed in terms of the HB grading system. The baseline lacrimation was analyzed based on the difference in moisture between both eyes (in $\mathrm{mm}$ ), which was obtained from the Schirmer I test. To analyze the deficits in taste sensation, we gave the patients with all modalities intact a score of 0 , those who lost 1 modality a score of 1 , those who lost 2 modalities a score of 2 , and those who lost all modalities a score of 3 .

Due to the sample size, we tested the normality of the data by using the Shapiro-Wilk test. The $\mathrm{p}$ values were 0.02 for the grade of facial nerve palsy and 0.001 for the difference in lacrimation. Accordingly, the alternative hypothesis had been accepted, signifying that the data were not normally distributed.

We tested the significance of the postoperative new deficits in taste (scores ranged from 0 for preservation of all three taste modalities to 3 for complete loss) and in lacrimation (the decrease in moisture on the side of surgery, in $\mathrm{mm}$ ) by using the Wilcoxon signed-rank test. Correlations between the taste score and lacrimation dysfunction and the grade of facial nerve palsy (HB grading system) were tested using Spearman's correlation test. Statistical significance was accepted at $\mathrm{p}$ values less than 0.05 .

\section{The Outcome of Taste and Lacrimation After Surgery}

Abnormality in taste perception was experienced by 1 patient $(3.3 \%)$ before surgery, and $6(20 \%)$ patients were newly affected after surgery. The disturbance of taste sensation was significantly higher after surgery $(p=0.02$, Wilcoxon signed-rank test). The baseline lacrimation decreased significantly (in $\mathrm{mm}$, according to the Schirmer I test) after surgery on the treated side $(\mathrm{p}<0.001$, Wilcoxon signed-rank test).

\section{Correlation Between the NI Dysfunction and the Postoperative Grade of Facial Nerve Palsy}

The disturbance in the taste sensation did not show a significant correlation with the grade of facial nerve palsy, and could happen in patients with normal postoperative functions of the motor portion of the facial nerve. The difference in lacrimation between the healthy side and the side of surgery showed a significant correlation with the grade of facial nerve palsy (coefficient $=0.85$, significant at $\mathrm{p}<0.001)$.

\section{Discussion}

The NI can be endangered during VS surgery due to its location along the motor fibers of the facial nerve. In normal NI, $20 \%$ of fibers are unmyelinated. Therefore, the sensory nerve fibers have less protection from mechanical trauma than do motor fibers. ${ }^{1,8,15,22}$ The disturbances to functions of the nonmotor portions of the facial nerve after VS surgery or radiosurgery have been sporadically reported in the literature..$^{13,14,16,20,21,24}$ In this study, we present a prospective analysis of the clinical aspects of the dysfunctions of the nonmotor portion of the facial nerve after VS surgery.

Schirmer's test showed that the baseline lacrimation was lower on the side of surgery in 23 patients (76\%). This decrease in lacrimation after surgery on the treated side, in comparison to the healthy side and to the preoperative baseline lacrimation on the side of surgery, was statistically significant. There was no change in the baseline lacrimation on the healthy side. The differences between the healthy side and the side of surgery showed a high correlation with the grade of the facial palsy. Irving et al. found that an absence or significant reduction of the production of tears was noted in $4 \%$ of patients before surgery and in $72 \%$ after surgery. The reduction was more likely to happen in patients with facial palsy. ${ }^{8}$ Incidences of crocodile tears are reported to range from $10 \%$ to $40 \%{ }^{8,12,20}$ In this series, only 1 patient (3\%) suffered from temporary crocodile tears. Another less reported symptom was epiphora (watery eye), which can happen due to the deficit pumping function of the orbicularis oculi muscle as well as the decreased tone of this muscle and flaccid lower eyelid in cases of facial palsy. ${ }^{11}$ Epiphora was reported by 4 patients $(13.3 \%)$ in this series and was resolved in 3 patients after 6 months with the recovery of the facial nerve. The fourth patient recovered after 1 year. The correlation between the facial nerve and lacrimation is complex. The decreased blinking associated with facial nerve palsy can cause dry eye. Dry eye is also caused by decreased lacrimal secretion due to the effect on the parasympathetic part of the facial nerve. Orbicularis oculi muscle plays a role in pumping the lacrimal secretion from the lacrimal sac to the nasolacrimal duct. These intermingling functional correlations can lead to a picture of a patient who suffers from the feeling of dry eye or foreign body (decreased/ insufficient blinking and spreading of the tears on the cornea), and decreased baseline lacrimal secretion together with epiphora (decreased pumping of the orbicularis oculi muscle and decreased muscle tone in the lower eyelid).

The disturbances in taste did not show a significant correlation with the degree of facial nerve palsy. After surgery, 2 patients $(6.6 \%)$ lost taste sensation on the anterior two-thirds of the tongue on the treated side. One of them did not suffer from disturbances of functions of the motor portion of the facial nerve or from lacrimation problems. The second patient had Grade III facial palsy. On the other hand, 1 patient in this study suffered from Grade IV facial palsy, and he did not suffer from disturbed taste sensation after surgery. Four patients (13.3\%) had decreased taste sensation on the side of surgery and recovered after 6 months. They had HB Grades II and III facial palsy. The bitter taste was not tested because bitter taste is perceived by the foliate papillae, whose signals are conveyed by the glossopharyngeal nerve, ${ }^{8,24}$ and accordingly we excluded 
the bitter sensation from the analysis. The disturbance of taste sensation in patients with VS surgery ranged in the literature from $22 \%$ to $48 \%{ }^{8}$ Watanabe et al. found that the number of patients with taste disturbance increased only slightly after surgery compared with before surgery (from $28.7 \%$ to $34.3 \%$ ), but the increase was not statistically significant. ${ }^{24}$ The correlation between the grade of facial nerve palsy and disturbance in taste function showed a discrepancy in the literature: Watanabe et al. and Irving et al. did not find a correlation, but Stripf et al. reported a positive correlation..$^{20}$ Nevertheless, it is worth mentioning here that Stripf et al. reported about the NI dysfunction and did not specify the disturbance in taste sensation.

In the current study, after 6 months the baseline lacrimation and the symptom of dry eye were resolving, corresponding to the recovery from the facial nerve palsy. Only 1 patient reported the symptoms of dry eye and decreased lacrimation on Schirmer's test after 1 year. This patient reported the feeling of dry eye after 24 months, whereas the facial palsy improved to HB Grade II and Schirmer's test showed no differences between sides. Although the baseline lacrimal secretion resolved, the deficient blinking could have been responsible for the persistent symptoms. The recovery from epiphora corresponded to the recovery of the functions of the motor portion of the facial nerve. Recovery from the partial loss of the taste sensation (in 4 patients) occurred in the first 6 months after surgery. Complete loss of the taste sensation did not resolve over a follow-up period of up to 2 years. Irving et al. reported that recovery of lacrimation and resolution of the symptoms of dry eye occurred in patients with good facial motor function (Grades I-III). Overall the rate of recovery of normal tearing was $27 \%$, with a mean time to recovery of 7 months. Normal taste sensation was recovered, however, in $42 \%$ of the affected cases, with a mean time to recovery of 7 months. ${ }^{8}$ On the other hand, Stripf et al. reported that recovery from tearing symptoms took 4.1 months. ${ }^{20}$ Stripf at al. assumed that the high degree of improvement in this symptom several months postoperatively might be explained by adaptation as well as the intact gustatory function of the other half of the tongue. ${ }^{23}$ In the series of Watanabe et al., the deficits in taste sensation resolved in $64.9 \%$ of the affected cases within 1 year. ${ }^{24}$ There was no quantitative analysis of the deficits and their resolution because most of these studies were based on questionnaires.

NI deficits could be subjectively recognized by the patients - 2 patients with complete loss of the taste sensation on the side of surgery and 2 patients with partial loss of taste complained about their symptoms. All patients with decreased baseline lacrimation and facial palsy reported symptoms of dry eye. However, the subjective feeling of dry eye persists even after normalization of baseline lacrimation if a degree of facial palsy persists, perhaps due to a decrease in the frequency or efficiency of blinking that is associated with facial palsy.

Although parasympathetic innervation of the nasal mucosa and the salivary glands is carried via the NI, none of our patients reported abnormally increased or decreased nasal or salivary secretion.

Our patients in this series were treated via the retrosigmoid approach. The lacrimation was affected in $76 \%$ of the patients and the taste sensation was affected in $20 \%$. Other approaches could have different incidences of an effect on the NI after VS resection. Stripf et al. reported that the middle fossa approach is associated with a higher incidence of lacrimation disturbances (78\%) and a lower incidence of taste disturbances (41\%), indicating the possibility of isolated injury of the greater petrosal nerve if the middle fossa approach was used. ${ }^{20}$

Recently, the patients' quality of life gained attention when investigators reported about the outcome after VS surgery. The available approaches for evaluation of the quality of life depend on a self-assessment questionnaire that is mailed to the patients. Questionnaires like the 36-Item Short Form Health Survey (SF-36), the 10-Item Patient-Reported Outcomes Measurement Information System short form (PROMIS-10), or the Glasgow Benefit Inventory (GBI) did not specify symptoms related to the NI dysfunction. ${ }^{5}$ A disease-specific questionnaire like the Penn Acoustic Neuroma Quality-of-Life Scale considered only the lacrimation dysfunction..$^{23}$ Stripf et al. developed a valuable questionnaire considering only the NI dysfunctions,${ }^{20}$ but it lacks objective evaluation and depends only on patient self-assessment.

The HB grading system of facial nerve palsy is very efficient for reporting about the function of the motor portion of the facial nerve. We recommend, however, adding the following categories: 1) gustatory/taste disturbance (G0: no disturbance, G1: decreased sensation or partial loss, G2: lost sensation); 2) dry eye (D0: absence of the feeling of dry eye, D1: feeling of dry eye with normal Schirmer test, D2: basal lacrimation on the affected side $<8 \mathrm{~mm}$ lower than the healthy side, D3: basal lacrimation in the affected side is $\geq 8 \mathrm{~mm}$ lower than the healthy side); and 3) presence or absence of epiphora (E0: no epiphora, E1: presence of epiphora) to the usual HB grading system (Table 3). Patients who get grade $\mathrm{G} 2$ or D3 will have a low chance of recovery from their symptoms within 2 years. Accordingly, we suggest reporting (for example, about our patient who suffered from Grade IV facial palsy postoperatively) as facial nerve dysfunction HB Grade IV, G0, D3, E1. A second

TABLE 3. Grading of NI dysfunctions after resection of VS

\begin{tabular}{lc}
\hline \multicolumn{1}{c}{ Dysfunction } & Grade \\
\hline $\begin{array}{l}\text { Gustatory/taste disturbance } \\
\text { No disturbance }\end{array}$ & $\mathrm{G} 0$ \\
\hline Decreased sensation of 1 or 2 modalities & $\mathrm{G} 1$ \\
\hline $\begin{array}{l}\text { Lost sensation } \\
\text { Dry eye }\end{array}$ & $\mathrm{G} 2$ \\
\hline $\begin{array}{l}\text { Absence of the feeling of dry eye } \\
\text { Feeling of dry eye w/ normal Schirmer test results }\end{array}$ & $\mathrm{D} 1$ \\
\hline $\begin{array}{l}\text { Baseline lacrimation on the affected side }<8 \mathrm{~mm} \text { lower than } \\
\text { the healthy side }\end{array}$ & $\mathrm{D} 2$ \\
\hline $\begin{array}{l}\text { Baseline lacrimation on the affected side } \geq 8 \mathrm{~mm} \text { lower than } \\
\text { the healthy side }\end{array}$ & $\mathrm{D} 3$ \\
\hline $\begin{array}{l}\text { Epiphora } \\
\text { Absence }\end{array}$ & $\mathrm{E} 0$ \\
\hline \begin{tabular}{l} 
Presence \\
\hline
\end{tabular}
\end{tabular}


example could be our patient who did not have palsy of the motor portion of the facial nerve, but who suffered from lost taste sensation on the side of surgery (HB Grade I, G2, D0, E0). Including the NI dysfunctions in the quality-oflife questionnaires and using the suggested grading system requires further validation from multiple centers and under different therapeutic approaches.

\section{Conclusions}

The NI can be affected after VS surgery. The disturbance of baseline lacrimal secretion was correlated with dysfunction of the motor portion of the facial nerve. However, the disturbance of the taste sensation was not correlated with the grade of facial nerve palsy. NI dysfunctions can be subjectively recognized by the patients. NI dysfunctions should be evaluated and separately reported after the management of VS.

\section{Acknowledgments}

We thank Mrs. Rica Mulamoottil and our neurophysiology team for their support and effort while performing the tests.

\section{References}

1. Alfieri A, Fleischhammer J, Peschke E, Strauss C: The nervus intermedius as a variable landmark and critical structure in cerebellopontine angle surgery: an anatomical study and classification. Acta Neurochir (Wien) 154:1263-1268, 2012

2. Alfieri A, Fleischhammer J, Prell J: The functions of the nervus intermedius. AJNR Am J Neuroradiol 32:E144-E145, 2011

3. Alfieri A, Strauss C, Prell J, Peschke E: History of the nervus intermedius of Wrisberg. Ann Anat 192:139-144, 2010

4. Boessert P, Grüttner C, van Ewijk R, Haxel B: [Changes in taste ability in patients with vestibular schwannoma.] Laryngorhinootologie 93:450-454, 2014 (Ger)

5. Carlson ML, Tveiten OV, Driscoll CL, Goplen FK, Neff BA, Pollock BE, et al: Long-term quality of life in patients with vestibular schwannoma: an international multicenter crosssectional study comparing microsurgery, stereotactic radiosurgery, observation, and nontumor controls. J Neurosurg 122:833-842, 2015

6. Gerganov VM, Klinge PM, Nouri M, Stieglitz L, Samii M, Samii A: Prognostic clinical and radiological parameters for immediate facial nerve function following vestibular schwannoma surgery. Acta Neurochir (Wien) 151:581-587, 2009

7. House JW, Brackmann DE: Facial nerve grading system. Otolaryngol Head Neck Surg 93:146-147, 1985

8. Irving RM, Viani L, Hardy DG, Baguley DM, Moffat DA: Nervus intermedius function after vestibular schwannoma removal: clinical features and pathophysiological mechanisms. Laryngoscope 105:809-813, 1995

9. Landis BN, Welge-Luessen A, Brämerson A, Bende M, Mueller CA, Nordin S, et al: "Taste Strips" - a rapid, lateralized, gustatory bedside identification test based on impregnated filter papers. J Neurol 256:242-248, 2009

10. Li N, Deng XG, He MF: Comparison of the Schirmer I test with and without topical anesthesia for diagnosing dry eye. Int J Ophthalmol 5:478-481, 2012

11. Madge SN, Malhotra R, Desousa J, McNab A, O'Donnell B, Dolman P, et al: The lacrimal bypass tube for lacrimal pump failure attributable to facial palsy. Am J Ophthalmol 149:155-159, 2010

12. Nakamizo A, Yoshimoto K, Amano T, Mizoguchi M, Sasaki
T: Crocodile tears syndrome after vestibular schwannoma surgery. J Neurosurg 116:1121-1125, 2012

13. Noonan KY, Rang C, Callahan K, Simmons NE, Erkmen K, Saunders JE: Nervus intermedius symptoms following surgical or radiation therapy for vestibular schwannoma. Otolaryngol Head Neck Surg 155:657-662, 2016

14. Park SH, Lee KY, Hwang SK: Nervus intermedius dysfunction following Gamma Knife surgery for vestibular schwannoma. J Neurosurg 118:566-570, 2013

15. Rhoton AL Jr, Kobayashi S, Hollinshead WH: Nervus intermedius. J Neurosurg 29:609-618, 1968

16. Sahu RN, Behari S, Agarwal VK, Giri PJ, Jain VK: Taste dysfunction in vestibular schwannomas. Neurol India 56:42-46, 2008

17. Samii M, Gerganov V, Samii A: Improved preservation of hearing and facial nerve function in vestibular schwannoma surgery via the retrosigmoid approach in a series of 200 patients. J Neurosurg 105:527-535, 2006

18. Samii M, Matthies C: Management of 1000 vestibular schwannomas (acoustic neuromas): the facial nerve-preservation and restitution of function. Neurosurgery 40:684695, 1997

19. Samii M, Matthies C, Tatagiba M: Management of vestibular schwannomas (acoustic neuromas): auditory and facial nerve function after resection of 120 vestibular schwannomas in patients with neurofibromatosis 2. Neurosurgery 40:696-706, 1997

20. Stripf T, Braun K, Gouveris H, Stripf EA, Mann WJ, Amedee RG: Influence of different approaches to the cerebellopontine angle on the function of the intermediate nerve. J Neurosurg 107:927-931, 2007

21. Tamura M, Murata N, Hayashi M, Régis J: Injury of the lacrimal component of the nervus intermedius function after radiosurgery versus microsurgery. Neurochirurgie 50:338344, 2004

22. Tubbs RS, Steck DT, Mortazavi MM, Cohen-Gadol AA: The nervus intermedius: a review of its anatomy, function, pathology, and role in neurosurgery. World Neurosurg 79:763767, 2013

23. van Leeuwen BM, Herruer JM, Putter H, Jansen JC, van der Mey AG, Kaptein AA: Validating the Penn Acoustic Neuroma Quality Of Life Scale in a sample of Dutch patients recently diagnosed with vestibular schwannoma. Otol Neurotol 34:952-957, 2013

24. Watanabe K, Saito N, Taniguchi M, Kirino T, Sasaki T: Analysis of taste disturbance before and after surgery in patients with vestibular schwannoma. J Neurosurg 99:9991003, 2003

\section{Disclosures}

The authors report no conflict of interest concerning the materials or methods used in this study or the findings specified in this paper.

\section{Author Contributions}

Conception and design: Metwali, Samii. Acquisition of data: Metwali, Kniese, Kardavani. Analysis and interpretation of data: Metwali, Kniese, Kardavani, Gerganov. Drafting the article: Metwali, Kniese, Gerganov. Critically revising the article: Gerganov, Samii. Reviewed submitted version of manuscript: Metwali, Kniese, Gerganov, Samii. Approved the final version of the manuscript on behalf of all authors: Metwali. Statistical analysis: Metwali. Study supervision: Samii.

\section{Correspondence}

Hussam Metwali: International Neuroscience Institute, Hannover, Germany.drhussamm@yahoo.com. 